\title{
Excitation of Gravity-Mode Pulsations in DA \& DB White Dwarfs
}

\author{
Yanqin $\mathrm{Wu}$ \\ Astronomy Unit, School of Mathematical Sciences, Queen Mary and \\ Westfield College, Mile End Road, London E1 \&NS, UK
}

\begin{abstract}
Oscillations in white dwarfs of hydrogen or helium envelopes are believed to be excited close to the surface, where convective energy transport dominates the stellar luminosity. The convective motion in these stars is fast and can respond instantaneously to the pulsation state. In this limit, we find the convective envelope to be the seat of mode excitation because it acts as an insulating blanket with respect to the perturbed flux that enters it from below. This retaining of the flux leads to driving. Driving exceeds radiative damping provided $\omega \tau_{\mathrm{c}} \geq 1$, where $\omega$ is the radian frequency of the mode and $\tau_{\mathrm{c}} \approx 4 \tau_{\text {th }}$ with $\tau_{\text {th }}$ being the thermal time constant evaluated at the base of the convective envelope. We follow Brickhill (1991) in naming this mechanism as 'convective driving'. We also studied the dynamical interaction between turbulent convection and pulsation. In the limit of fast convection, turbulent damping inside the convective region is negligible, while that coming from the overshoot region is more significant. I discuss the application of 'convective driving' in other types of pulsating stars.
\end{abstract}

\section{Introduction}

Along the white dwarf cooling sequence, there are three locations inside which white dwarfs are observed to exhibit gravity-mode pulsation. We are concerned with the two instability strips at lower temperature, DA (with hydrogen atmosphere, also named ZZ Ceti) and DB (helium atmosphere).

Difficulty in explaining pulsation driving in these two populations of pulsators is related to their surface convection zones. These convection zones are formed when the white dwarfs are cool enough to have hydrogen (or helium in the case of DBs) partially recombining in their atmosphere and have consequently enhanced opacity.

The partial ionization zones lie too high up in the atmosphere to be able to drive the gravity-modes via the $\kappa$-mechanism. However, the thermal time at the bottom of these convection zones typically range from a few seconds to a few hundred seconds, falling in the same range as the observed g-mode periods (from a hundred to a thousand seconds). Perhaps the convection zone is the driving region for the observed g modes?

To answer this question, one needs to take convection-pulsation interaction fully into account. Firstly, the high matter density in the white dwarf atmo- 


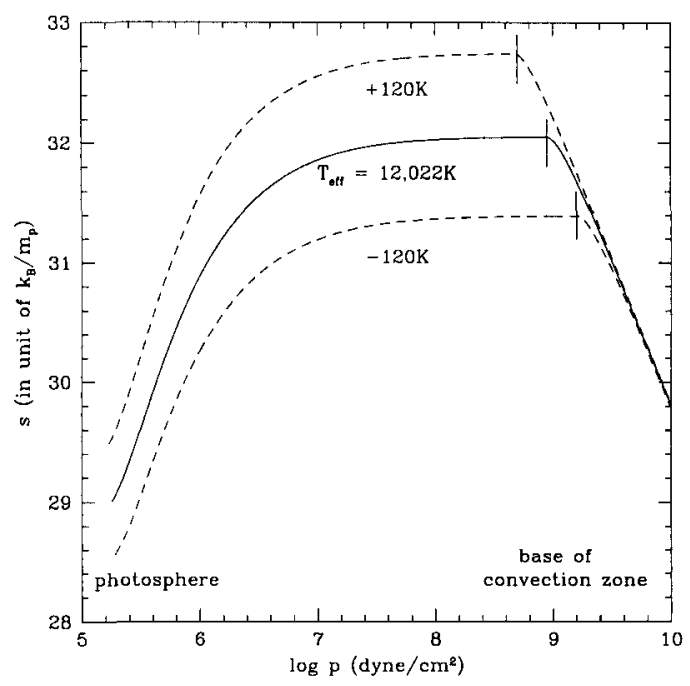

Figure 1. Entropy profiles as functions of logarithmic pressure in the upper atmosphere for three adjacent white dwarf models. The bottom of the convection zone is indicated by a vertical bar in each model. We construct these models based on mixing length theory (see Wu \& Goldreich 1999).

spheres implies that convection carries most of the stellar flux, radiative flux is insignificant in the driving region. Secondly, convection in these stars is fast, with the convective turn-over time typically of order a second or less.

Following the early studies by Brickhill $(1983,1991)$ and Gautschy et al. (1996), we (Goldreich \& Wu 1999a; Wu \& Goldreich 1999) carried out both quasiadiabatic and nonadiabatic studies into the problem of gravity-mode excitations in fast convection zones. We found the fast convection zones in DA and $\mathrm{DB}$ variables are responsible for exciting low to medium radial order gravitymodes.

\section{Convective Driving}

This 'convective driving' can be best illustrated by investigating the entropy variations in the convection zone during pulsation. This is shown in Figure 1.

Consider a star at equilibrium $T_{\text {eff }}=12,022 \mathrm{~K}$, entropy in its convection zone is depicted by the solid middle curve in Figure 1. Most of the white dwarf convection zone is isentropic, except in the partial ionization region below the photosphere where it is superadiabatic. This latter region has low material density and high opacity.

When the surface convection zone is perturbed by pulsation, e.g., when it gets warmer by $120 \mathrm{~K}$, its entropy rises, roughly to the dashed curve marked 
by ' $+120 \mathrm{~K}$ ' in Figure $1 .{ }^{1}$ This is characteristic of an instantaneously adjusting convection zone. Fast eddy turn-over time dictates that most of the convection zone remain isentropic during the whole pulsation cycle. When the photosphere becomes warmer, ionization fraction increases, and this brings about higher photospheric entropy. At the same time, the entropy gradient in the superadiabatic layer becomes steeper to allow a larger convective flux to pass through. These two factors, together with the fact that most of the convection zone remains isentropic during pulsation, lead to entropy rise when the convection zone is warmer. We find the behaviour of the convection zone during different phases of the pulsation can be well described by equilibrium stellar models that are slightly different in their effective temperatures, as in Figure 1.

As argued above, the convection zone absorbs heat (rise in entropy) when it is warmer, and loses heat when cooler. The resulting Carnot cycle feeds back positively towards the pulsation, i.e., stellar luminosity is converted to pulsation energy by the instantaneously readjusting convection zone. We follow Brickhill (1991) in naming this driving as 'convective driving'. Figure 2 depicts the nonadiabatic eigenfunction (in terms of flux variations) and the work integrand calculated for a g-mode of period $430 \mathrm{~s}$, with spherical degree $\ell=1$ in a star of $T_{\text {eff }}=12,000 \mathrm{~K}^{2}$ Flux variation at the photosphere $\left((\delta F / F)_{\mathrm{ph}}\right)$ is reduced in magnitude and delayed in phase compared with that at the radiative-convective boundary $\left((\delta F / F)_{\mathrm{b}}\right)$. This results from the entropy variations inside the convection zone, as is necessary for driving. The two flux variations are related to each other as (for derivations, see Goldreich \& Wu 1991a)

$$
\left(\frac{\delta F}{F}\right)_{\mathrm{ph}}=\frac{1}{1-i \omega \tau_{\mathrm{c}}}\left(\frac{\delta F}{F}\right)_{\mathrm{b}}
$$

where $\tau_{c}$ is the thermal time scale that describes the adjustment of the convection towards pulsation. It is roughly 4 times the conventional thermal time measured at the bottom of the convection zone. As the surface visibility depends on the depth of the convection zone, a time-varying convection zone depth (due to pulsation, see Fig. 1) necessarily distorts the original sinusoidal light-curves. This is likely the origin of the 'combination frequencies' observed in many white dwarfs (Brickhill 1992; Wu 1997).

As is shown by Figure 2, the work integrand is at positive maximum in the bottom scale height of the convection zone, indicating where most of the driving comes from. The photosphere and the superadiabatic layer determine the sign of the entropy variation during pulsation, while the main convection zone acts as a long lever arm that provides most of the driving. The underlying radiative region is dissipative to pulsation, as the opacity there requires the heat to be lost when the region rises in temperature. Overall overstability of a $\mathrm{g}$ mode is attained when the convective driving exceeds the radiative damping. Analytically, we find this to be summarised by an overstability criterion,

$$
\omega \tau_{c} \geq \text { constant. }
$$

\footnotetext{
${ }^{1}$ We ignore changes in the depth of the convection zone in our stability analysis.

${ }^{2}$ The stellar models are kindly provided us by Bradley (1996).
} 


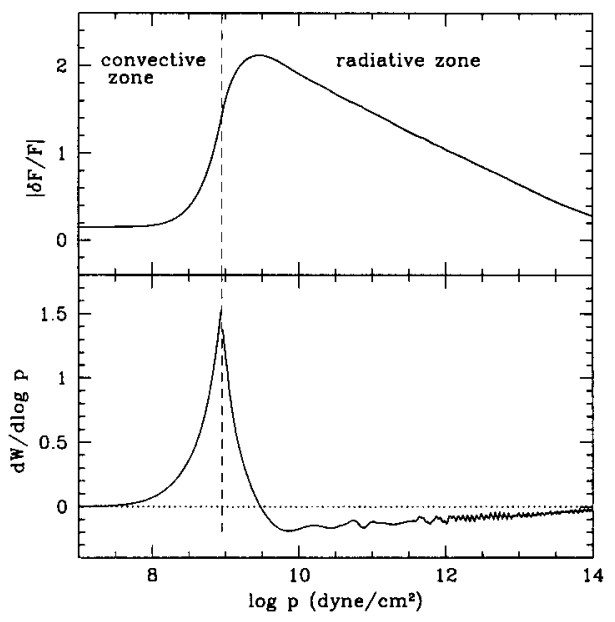

Figure 2. The nonadiabatic eigenfunction (shown as the modulus of the fractional flux variations) and work integrand for an $\ell=1$ mode with a period of $430 \mathrm{~s}$. Both quantities are in arbitrary units and are plotted against $\log p$. The stellar model has $T_{\text {eff }}=12,000 \mathrm{~K}$, and has a convection zone extending downwards to $\log p=8.95$ in ML2. The thermal time at the bottom of this zone is $200 \mathrm{~s}$.

The constant is roughly unity for variable white dwarfs, except in the coolest variables where this constant rises to of order ten. ${ }^{3}$ Cooler stars have deeper convection zones and are therefore capable of exciting longer period g-modes. This trend of longer period pulsations in cooler stars has been observed and is summarised by Clemens (1993) for DA variables.

Nonadiabatic results on g-mode overstability are collectively shown in Figure 3. For a mixing length description that is close to ML2, we find the blue edge of the DA instability strip lies at $T_{\text {eff }}=12,700 \mathrm{~K}$. This is when the convection zone first becomes thick enough during white dwarf cooling that its driving effect to the $k=1, \ell=1 \mathrm{~g}$-mode exceeds the damping effect from the radiative region below. A more efficient mixing length model will raise the temperature of the blue edge.

It is an interesting question as to what gives rise to the observed red edge of the instability strip. We found the following two issues conspire to result in a red edge at around $T_{\text {eff }}=11,700 \mathrm{~K}$ (ML2 prescription for convection). The first is surface visibility. Equation 1 indicates all modes with $\omega \tau_{c} \gg 1$ are relatively invisible at the surface, while nonadiabatic calculations find these modes to be the only unstable ones in such cool stars. The second is the maximum amplitudes overstable modes can achieve. We found ( $\mathrm{Wu} \&$ Goldreich, in preparation)

\footnotetext{
${ }^{3}$ This is a result of the enhanced radiative dissipation when the propagating region of $g$-modes is modified by the deepening convection zone.
} 


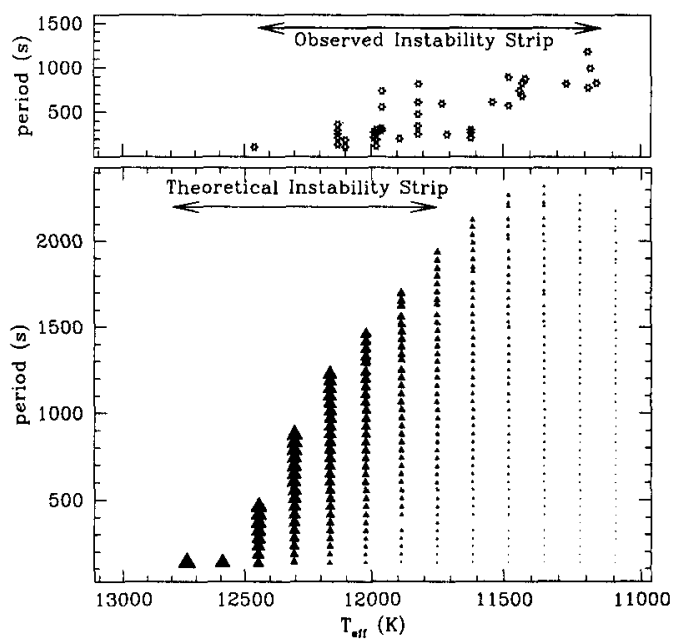

Figure 3. Observational and theoretical instability strips for DA white dwarfs. The upper panel displays each of the known ZZ Ceti stars according to the dominant period in its pulsation spectrum (Bradley 1995), and its inferred effective temperature (Bergeron et al. 1995). The lower panel shows the panoply of overstable $\ell=1$ gravity-modes based on fully nonadiabatic calculations (Wu \& Goldreich 1999, see also that article for details on the stellar models). Each overstable mode is marked with a solid triangle whose size is proportional to the diminution factor of the flux perturbation in the convection zone, $\left[1+\left(\omega \tau_{\mathrm{c}}\right)^{2}\right]^{-1 / 2}$ (eq. 1). When comparing observational and theoretical instability strips, one should bear in mind that both depend upon the assumed mixing-length.

that, close to the above temperature, virtually all excited modes are nonlinearly saturated at small amplitudes such as to escape detection. Both mode resonance and shear turbulence are responsible for the saturation.

Goldreich \& Wu (1999b) considered the problem of dynamic interaction between convection and g-mode pulsation. The main conclusion from this work is that the fast turn-over time in the convective region requires almost shear-less horizontal movement in the pulsation, and consequentially, there is little turbulent damping arising from the convection zone proper. However, we did find more complicated behaviour in the interface between convection and radiation which may have interesting implications for observations.

\section{3. $\gamma$ Doradus Stars}

A newly discovered class of variable stars, the $\gamma$ Doradus variables (Kaye et al. 2000), share some of the same characteristics as the DA and DB white dwarfs discussed here. Surface convection sets in these F-type main-sequence 
stars. In the models for these stars, the eddy turn-over time scale is short ( $\simeq 0.1$ day) compared to the periods of the observed g-mode pulsation $(0.3$ to 5 days). The He I partial ionization region is engulfed by the convection zone, and $\kappa$-mechanism is likely to be insignificant. Adopting the instantaneous assumption for convection-pulsation interaction, we find these stars can excite a number of gravity-modes that have periods corresponding to the observed ones.

\section{References}

Bergeron, P., Wesemael, F., Lamontagne, R., Fontaine, G., Saffer, R. A., \& Allard, N. F. 1995, ApJ, 449, 258

Bradley, P. A. 1995, Baltic Astronomy, 4, 536

Bradley, P. A. 1996, ApJ, 468, 350

Brickhill, A. J. 1983, MNRAS, 204, 537

Brickhill, A. J. 1991, MNRAS, 251, 673

Brickhill, A. J. 1992, MNRAS, 259, 519

Clemens, J. C. 1993, Baltic Astronomy, 2, 407

Gautschy, A., Ludwig, H. G., \& Freytag, B. 1996, A\&A, 311, 493

Goldreich, P. \& Wu, Y. 1999a, ApJ, 511, 904

Goldreich, P. \& Wu, Y. 1999b, ApJ, in press

Kaye, A. B., Handler, G., Krisciunas, K., Poretti, E., \& Zerbi F. M. 2000, in these proceedings, p. 426

Wu, Y. 1997, Ph.D. thesis, California Institute of Technology

Wu, Y. \& Goldreich, P. 1999, ApJ, 519, 783

\section{Discussion}

Hiromoto Shibahashi: I have two questions. The first one is, in your nonadiabatic calculations, how large is the contribution from the opacity-mechanism to the work integral? The second question is, is your theory applicable to the DO white dwarfs (PNNV stars)?

Yanqin Wu: In the work integral (Fig. 2), most of the driving comes from the bottom scale height of the convection zone. $\kappa$-mechanism does not function (directly) here as the strong convective response overrides the changes in the radiative flux. DO variable white dwarfs are not known to have surface convection zones, they are likely to be excited by a different mechanism than that for DA and $\mathrm{DB}$ variables.

Travis Metcalfe: Can you predict the (light curve) pulse shapes with this prescription for the driving mechanism?

Yanqin $W u$ : During pulsation, the convection zone varies in size, with a high $T_{\text {eff }}$ corresponding to a shallower convection zone. Combining this information with equation 1 , we find the emergent light curve is likely to have sharp ascents and shallow descents. This has been observed, so my answer is hardly a prediction. 